\title{
Does the medicating ADHD increase or decrease the risk for later substance abuse?
}

Attention deficit hyperactivity disorder (ADHD) onsets in early childhood and affects approximately $5 \%$ of juveniles persisting into adolescence in $75 \%$ of cases and into adulthood in up to one-half of cases. ${ }^{1}$ Untreated individuals with ADHD are at a two-fold risk for the development of nicotine and substance abuse (SA; including drug or alcohol abuse or dependence) through their life. ${ }^{2}$

What remains unclear is the role of ADHD treatment, in particular, pharmacotherapy, on the risk for SA. Given that the stimulants (methylphenidate and amphetamine) are the most commonly prescribed agents for ADHD, many parents, clinicians, and scientists have questioned whether exposure to this class of medication in ADHD youth could either lead to prescription abuse or serve as gateway to the abuse of other drugs. These concerns are based on the abuse liability of the stimulants in both preclinical animal and human studies. ${ }^{3}$

One manner to explore this important issue is to systematically review the ADHD literature to assess the link between SA and prior exposure to stimulants or other psychotropic medications. Using PubMed, a search of the literature was conducted for scientific abstracts and articles dealing with ADHD and risk for SA using both prospective and retrospective studies of children, adolescents, and adults with ADHD. This search revealed a total of seven studies in which children aged 7-9 years were exposed to stimulant treatment and followed up at least four years later including the United States and Germany. ${ }^{4}$ Overall, data from 766 treated subjects and 429 untreated subjects with ADHD were evaluated. Five of seven studies had similar severity of ADHD/comorbidity at baseline. In all of the studies, stimulants were the psychotropic used for ADHD.

Four studies were available commenting on ADHD pharmacotherapy and SA in adolescence. ${ }^{4}$ In a four year followup study from our group, medicated ADHD ( $\mathrm{N}=117)$, unmedicated ADHD $(\mathrm{N}=45)$, and unmedicated non-ADHD control youths $(\mathrm{N}=344)$ were evaluated in mid-adolescence. Replicating previous findings, baseline ADHD pharmacotherapy was strongly associated with lower rates of SA or dependence in mid-adolescence including alcohol, cocaine, stimulants, and hallucinogens compared to unmedicated ADHD youth. Rates of SA or dependence were comparable between the medicated ADHD and non-ADHD control groups. Molina and Pelham also observed lower rates of substance use disorders (SUD) in treated versus untreated ADHD youth at mid-adolescence. Adolescents treated pharmacologically $(\mathrm{N}=65)$ were at lower risk for SUD at five year followup compared to a group of ADHD adolescents not treated pharmacologically $(\mathrm{N}=73)$. Reductions in both alcohol and drug use disorders were noted in the treated group. Treated and untreated groups had similar ADHD severity and rates of disruptive disorders at baseline. Huss and colleagues presented preliminary information from a prospective, nonrandomized study of low dose, high dose, and no use of methylphenidate in youths with ADHD growing up $(\mathrm{N}=161)$ with SA as the main outcome. They reported that stimulant treatment was associated with reduced $\mathrm{SA}$, and that a more robust reduction was noted in the higher dose methylphenidate group.

There were five studies available on adults with ADHD who were treated pharmacologically as children and followed up in adulthood. ${ }^{4}$ For example, Lambert and colleagues reported that adults previously treated with stimulant medications $(\mathrm{N}=93)$ had statistically significant higher rates of cigarette and cocaine use compared to ADHD adults not previously stimulant treated $(\mathrm{N}=81)$. No differences were detected between groups on other drugs. The major limitation to the study includes apparent differences in baseline severity of illness between treatment groups. In other prospective, 15 year follow-up study of ADHD youth into adulthood, a lower risk for an alcohol use disorder, but not drug use disorder, was observed in adults treated $(\mathrm{N}=182)$ versus untreated $(\mathrm{N}=37)$ as youth with stimulant medications. Adults who were considered responders to stimulant medications were found to have lower rates of SUD than those considered nonresponders. Using a retrospective recall of past treatment in adults with ADHD, Huss recently reported a significantly reduced risk of SUD associated with treatment. In this study of 201 young adults, ADHD pharmacotherapy was associated was a significant 5.5-fold reduced risk of SUD.

Using metanalysis, we recently reported on the overall and specific effects of stimulant treatment on later SA. ${ }^{4}$ We found the pooled estimate of the odds ratio indicated a 1.9 fold reduction in risk for SA in youth treated with stimulants compared to youth not receiving pharmacotherapy for ADHD with a similar reduction in risk for later drug and alcohol use disorders. 
Additional analyses showed that the results could not be accounted for by any single study or by publication bias.

The results of these studies does not support the hypothesis of an increased risk for SA associated with ADHD pharmacotherapy; in contrast, the bulk of data support the hypothesis of a protective effect of medication treatment of ADHD on the development of later substance abuse.

Clarification of the critical influence of ADHD treatment in youth on later SA remains hampered by methodological issues. Since prospective studies in ADHD youth were largely naturalistic, and hence, not randomized at baseline to medication, attempts to disentangle positive or deleterious effects of treatment from the severity of the underlying condition(s) are hampered by serious confounds. In prospective studies in which baseline severity was similar between treated and untreated groups, there was a protective factor of pharmacotherapy on later substance use disorders. In contrast, in studies in which baseline severity was unequal between groups, ADHD pharmacotherapy was related to increased drug use and abuse-mostly likely related to the severity of the ADHD and comorbid conduct disorder at baseline and not the treatment effect. Moreover, the degree to which family history of SA, a strong predictor of SA in individuals was distributed between treatment groups across all studies is not known.

The mechanism by which ADHD pharmacotherapy may lead to reduce SA remains unclear and under study at our site. ADHD may lead to less impairing symptom and overall improvement in self esteem and academic and social success which are direct and indirect risk factors for SA. It may also be that the close monitoring of youth receiving medications may have had a protective effect on the development of SUD, independent of the actual medication effect.

Despite the many limitations of the literature in this area, the current data supports that ADHD pharmacotherapy, almost invariably with stimulants, does not increase, but appears to decrease the risk for SA. Further studies investigating the longerterm outcome of ADHD youth pharmacologically treated and later SA and the putative mechanisms of ADHD pharmacotherapy reducing SA are currently underway.

Timothy E Wilens

Clinical Research Program in Pediatric

Psychopharmacology, Massachusetts General Hospital and Harvard Medical School. Boston

\section{References}

1. Wilens TE, Biederman J, Spencer TJ. Attention deficit/hyperactivity disorder across the lifespan. Ann Rev Med 2002;53:113-31.

2. Biederman J, Wilens TE, Mick E, Milberger S, Spencer TJ, Faraone SV. Psychoactive substance use disorders in adults with attention deficit hyperactivity disorder (ADHD): effects of ADHD and psychiatric comorbidity. Am J Psychiatry 1995;152(11):1652-8.
3. Kollins SH, MacDonald EK, Cush CR. Assessing the abuse potential of methylphenidate in nonhuman and human subjects: a review. Pharmacol Biochemistry Behav 2001;68:611-27.

4. Wilens T, Faraone S, Biederman J, Gunawardene S. Does stimulant therapy of ADHD beget later substance abuse: A metanalytic review of the literature. Pediatrics 2003;11(1):179-85. 